\title{
Updated Review on Pathology of Endocervical Adenocarcinoma with Emphasis on Clinically Relevant Findings
}

\author{
Adela Cimic, Xiaolin Liu-Jarin \\ Department of Pathology and Cell Biology, Columbia University Irving Medical Center, New York, NY, United States \\ Correspondence: ac4620@cumc.columbia.edu; Tel.: + 1212342 3013; Fax.: + 12123051295
}

Received: 13 January 2021; Accepted: 18 February 2021

\begin{abstract}
In the present review, we summarize and critically appraise recent advances in the pathology of endocervical adenocarcinoma. In recent years, the diagnosis of endocervical adenocarcinoma has shifted from morphologic criteria classification in 2014 World Health Organization (WHO) to etiology- based classification of International endocervical adenocarcinoma criteria and classification (IECC). IECC recommends classifying endocervical adenocarcinoma into Human Papillomavirus (HPV)associated and non-HPV-associated. Ultimately, this approach may lead to different treatment options based on molecular pathways rather than purely based on the tumor's grade and stage. Recently, the College of American Pathologists (CAP) has incorporated stromal invasion patterns as an optional data set in the synoptic report. The pattern of invasion classification is a valuable prognostic tool in excision specimens. Conclusion: IECC is a simple classification system that recognizes and classifies endocervical tumors based on pathogenesis and association to HPV. The pathologists should also be familiar with the patternbased classification of endocervical adenocarcinoma.
\end{abstract}

Key Words: Endocervical Adenocarcinoma - HPV - Classification • Silva Patterns.

\section{Introduction}

Endocervical adenocarcinoma is a relatively rare disease but still accounts for approximately $25 \%$ of all cervical carcinomas (1). The most recent World Health Organization (WHO) classification (2014) classifies in the cervical adenocarcinomas based on morphologic findings (2). There are multiple recent advancements in cervical pathology to recognize and to keep in mind for practicing pathologists. In this review article, we will review the most recent changes in cervical pathology to keep the reader up to date.

In the first part, the article will focus on a relatively new classification. It is common knowledge that the high-risk human papillomavirus (HPV) is a primary culprit in developing endocervical adenocarcinoma. International endocervical ad- enocarcinoma criteria and classification (IECC) classifies endocervical adenocarcinomas into two major subtypes: 1. HPV-associated 2. Non-HPVassociated subtype. This classification incorporates etiology but also coincides with distinctive morphologic patterns and clinical findings (3). The second part will focus on morphologic and stromal invasion patterns and their clinical significance. Proposal for the Silva system for endocervical carcinoma was published in 2013, but it was recently integrated as an optional data set in the College of American Pathologists (CAP) synoptic report.

Lastly, we will briefly comment on changes implemented in the International Federation of Gynecology and Obstetrics (FIGO) staging classification system. 


\section{International Endocervical Adenocarcinoma Criteria and Classification (IECC): HPV-Associated Endocervical Adenocarcinoma}

HPV-associated endocervical adenocarcinoma has similar risk factors as cervical squamous cell carcinomas, including multiple sexual partners, young age at first intercourse, obesity, smoking etc. Most endocervical adenocarcinomas are HPV-associated and encompass about $85-90 \%$ of all endocervical adenocarcinomas (2). Three subtypes HPV 16, 18, and 45, appear to be the most prevalent and accounted for $94 \%$ of the cases (4). HPVassociated endocervical adenocarcinoma presents in the younger age ( $<50$ years), and most of them develop in the transformation zone similarly to cervical squamous cell carcinoma. The future treatments based on this classification may be etiology driven and may have better outcomes. The current treatment approach is that all endocervical adenocarcinomas are treated the same, stage by stage, with no specific treatment strategy based on genetic signature or etiology.

\section{Usual Type HPV-Associated Endocervical Adenocarcinoma}

Relatively good news is that most endocervical adenocarcinomas are easily recognizable as usualtype endocervical adenocarcinoma. The glands may have papillary, micropapillary, cribriform and single-cell pattern, but the distinctive cytologic features are at least focally present. Those features include pseudostratified, elongated, and enlarged nuclei with eosinophilic cytoplasm and many apical "floating" mitotic figures. Numerous apoptotic bodies are also easily identifiable (Figure 1). The principal differential diagnosis for usual-type endocervical adenocarcinoma is endometrioid adenocarcinoma. Immunohistochemistry (IHC) is very helpful in differentiating the two. While endocervical adenocarcinoma is HPV-associated and p16 strongly and diffusely positive, endometrioid adenocarcinoma is patchy positive for $\mathrm{p} 16$ with a high estrogen (ER) and progesterone (PR)

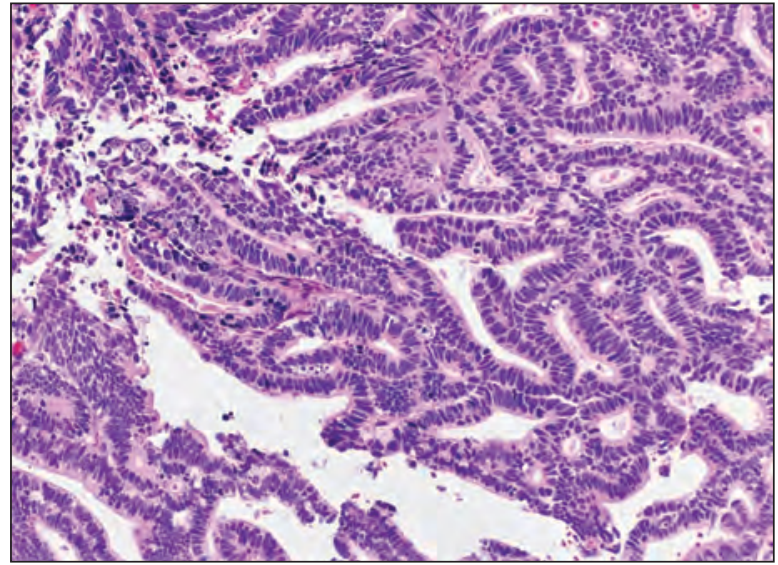

Figure 1. HPV-associated endocervical adenocarcinoma with numerous "floating mitosis" and apoptotic bodies. (Olympus BX43, 20×).

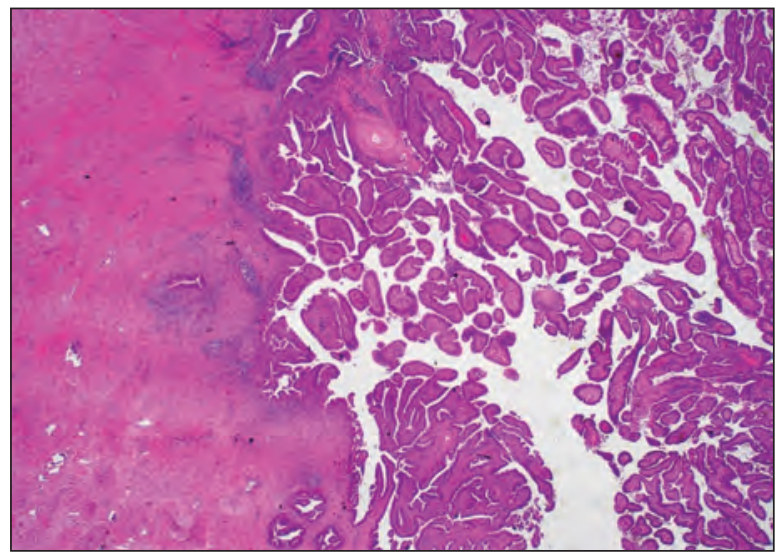

Figure 2. Villoglandular variant of endocervical adenocarcinoma in a 32-year-old woman. Clinically, a large mass with only superficial invasion. Please note that the invasive portion of the tumor is glandular. Eight years later, the patient is well without metastases or recurrence. (Olympus BX43, 2X).

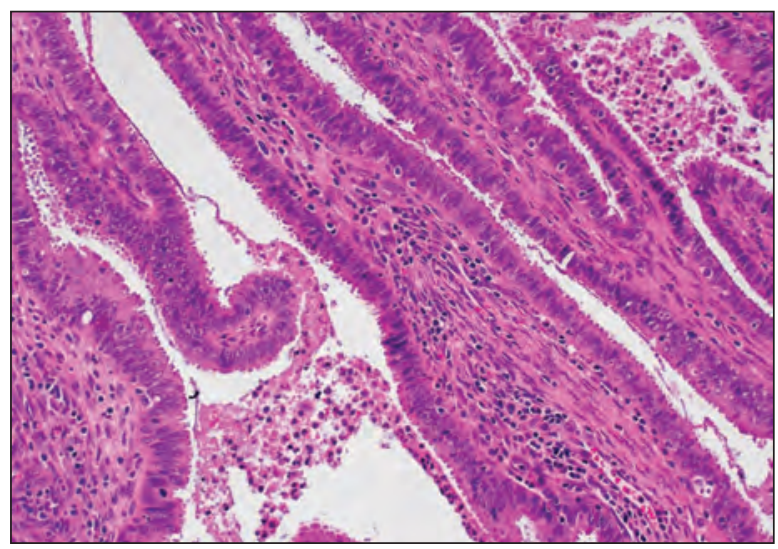

Figure 3. A high-power view reveals classical cytologic features of villoglandular endocervical adenocarcinoma. (Olympus BX43, 20x). 
receptor and vimentin positivity (5). While primary endometrioid adenocarcinoma may arise in endometriosis of the cervix, it is exceedingly rare. Preoperative communication with the clinical team and review of radiologic findings will help distinguish these two primary sites.

A rare but distinctive pattern worth mentioning is primary villoglandular endocervical adenocarcinoma. This subtype is recognized by long, thin exophytic papillae with cytologic features of usual-type endocervical adenocarcinoma (Figures 2 and 3). Invasion, if present, is usually superficial. Deeper components/invasive tumor will have glands rather than papillae. This variant is rarely associated with lymphovascular space invasion or lymph node metastasis and therefore carries a better prognosis $(6,7)$. A gynecologist can grossly visualize the tumors. They may present as a higher stage by the International Federation of Gynecology and Obstetrics (FIGO) staging, which would be treated with hysterectomy. However, if the biopsy suggests this variant, a cone biopsy may be an excellent initial treatment option to evaluate for stromal invasion. The cone excisional biopsy would be sufficient therapy for no or minimal invasion in this subtype of endocervical adenocarcinoma despite the clinical appearance of a larger tumor. This treatment modality should be considered for fertility preservation in young patients.

Additionally, a distinctive pattern is a micropapillary pattern. Microscopically it is characterized by small, cohesive papillary groups of neoplastic cells surrounded by stromal clefting. It is often associated with lymphovascular space invasion and lymph node metastasis $(8,9)$. The prognosis of this subtype is worse than pure usual type of endocervical adenocarcinoma. It is considered histology of aggressive behavior, and it can be associated with other subtypes (10).

\section{Mucinous Type HPV-Associated Endocervical Adenocarcinoma}

Mucinous endocervical adenocarcinomas are a diverse group of tumors. They are defined by the presence of intracellular mucin. The IACC classi- fication recognizes 1. Mucinous adenocarcinoma, not otherwise specified (NOS) 2. Intestinal type, and 3. Signet ring-type. To be classified as such, the tumors have to have $>50 \%$ of a tumor cell with intracytoplasmic mucin, intestinal goblet cell morphology, or signet cells, respectively. To be classified as HPV-associated, all mucinous types have at least a small morphologic component of usual type endocervical adenocarcinoma (3).

Another distinctive subtype and a relatively newly described entity is invasive stratified mucinous endocervical adenocarcinoma (iSMILE) (11). Histologic features are characteristic invasive nests of stratified columnar epithelium with hyperchromatic nuclei and variable amounts of intracytoplasmic mucin (Figures 4-5). Intracytoplasmic mucin may range from large intracellular droplets

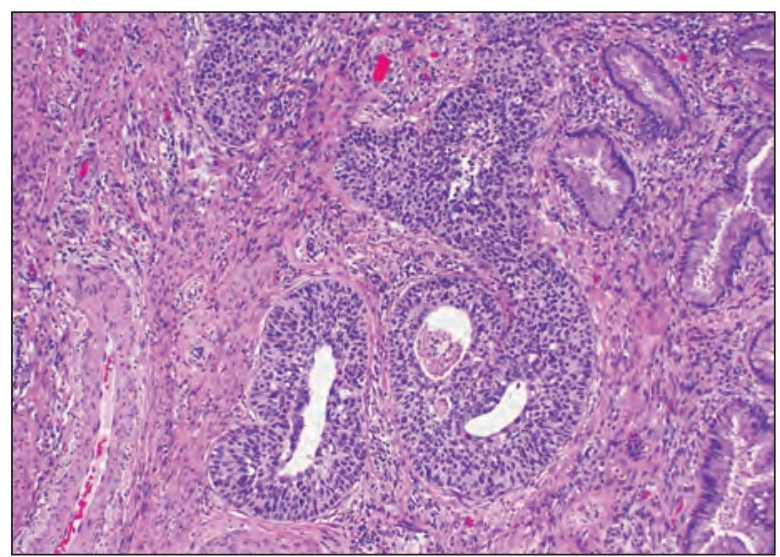

Figure 4. iSMILE nests of neoplastic cells lined with palisading cells at the periphery. (Olympus BX43, 10X).

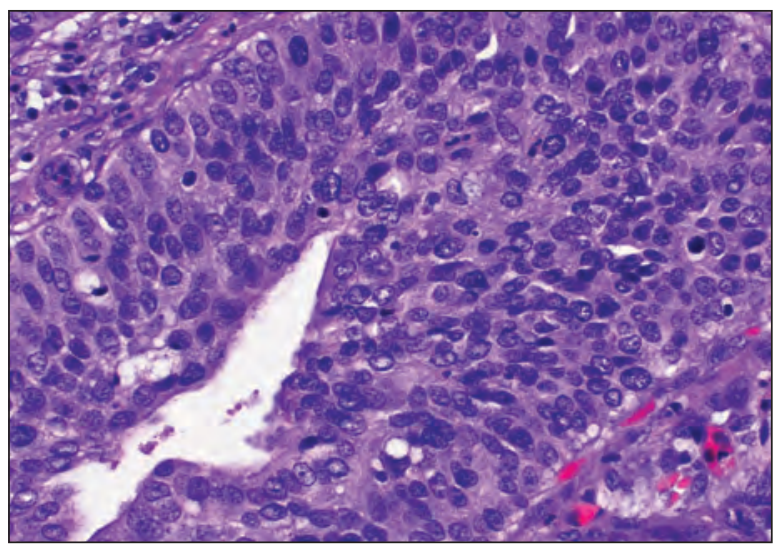

Figure 5. iSMILE mucin-poor variant can be difficult to distinguish on the biopsy material from glandular involvement. (Olympus BX43, 40X). 


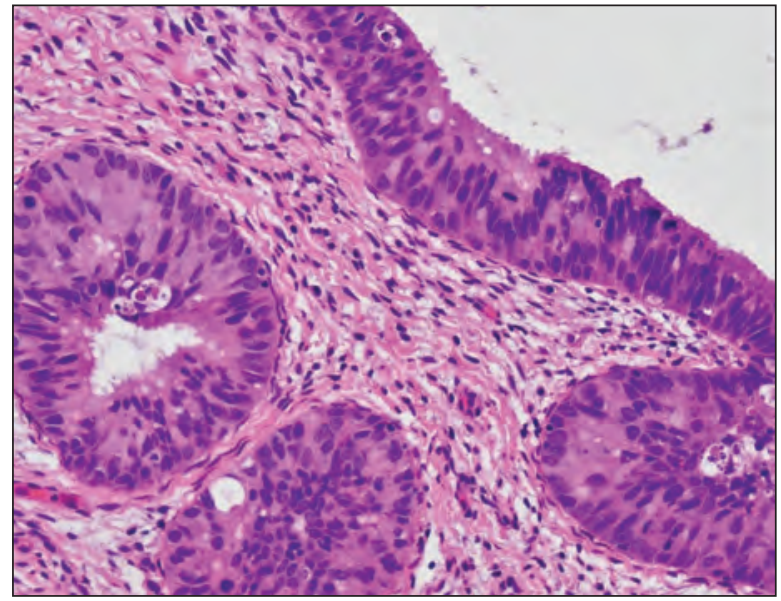

Figure 6. The very classical look of SMILE lesion with intracellular mucin in this stratified epithelium. Mitotic figures are readily identified. This entity is often coupled with endocervical adenocarcinoma in situ or squamous intraepithelial lesion. (Olympus BX43, 40X).

to almost entirely mucin depleted lesions. The nests at the periphery are lined by palisading cells that tend to be positive for $\mathrm{p} 40$ and $\mathrm{p} 63$. The tumor has a lower prevalence for PAX8 immunostaining with frequent nuclear expression of $\mathrm{p} 53$ by immunohistochemistry. The mucin-poor variant may be difficult to recognize, but the large nests with the characteristic immunohistochemistry support the diagnosis. This lesion often arises or is associated with histologically distinct intraepithelial lesionstratified mucinous intraepithelial lesion (SMILE) (Figure 6). This distinct entity is readily recognizable by multi-layered and stratified epithelium similar to the squamous intraepithelial lesion but intracytoplasmic mucin vacuoles. The lesion is positive for p16 by immunohistochemistry. The previously recognized adenosquamous carcinoma should be only diagnosed in the presence of unequivocal adenocarcinoma and squamous cell carcinoma component (12).

\section{International Endocervical \\ Adenocarcinoma Criteria and Classification (IECC): Non-HPV-Associated Endocervical Adenocarcinoma}

Non-HPV-associated endocervical adenocarcinoma has four subtypes. The most common is a gas- tric subtype, followed by clear cell, endometrioid, and mesonephric adenocarcinoma.

\section{Gastric Type Endocervical Adenocarcinoma}

Gastric type endocervical adenocarcinoma is a spectrum of lesions that ranges from well-differentiated to poorly differentiated adenocarcinomas (Figures 7-9). This type of adenocarcinoma usually occurs in older patients. It usually presents as a larger lesion and often with no history of positive HPV test or abnormal Pap test. Lobular cervical glandular hyperplasia (LCGH) has been suggested to be a precursor lesion. This adenocarcinoma has pale cytoplasm, distinct cytoplasmic borders, deep invasion, glands near blood vessels, and often pools of mucin. Gastric type mucin can be easily identified using Alcian blue/PAS stain or MUC6 immunohistochemistry staining (13). The pathogenesis is via HPV independent pathways driven by TP53, SDK 11, GNAS, and KRAS mutations (14). Immunohistochemistry profile is not specific, and many times the cells are positive for only for CK7. MUC6 and p53 immunoreactivity can be seen in almost half of the cases. PAX8 is frequently negative. Genetically, the associations have been made with Lynch (Hereditary non-polyposis colorectal cancer syndrome) and Peutz-Jeghers syndromes $(15,16)$.

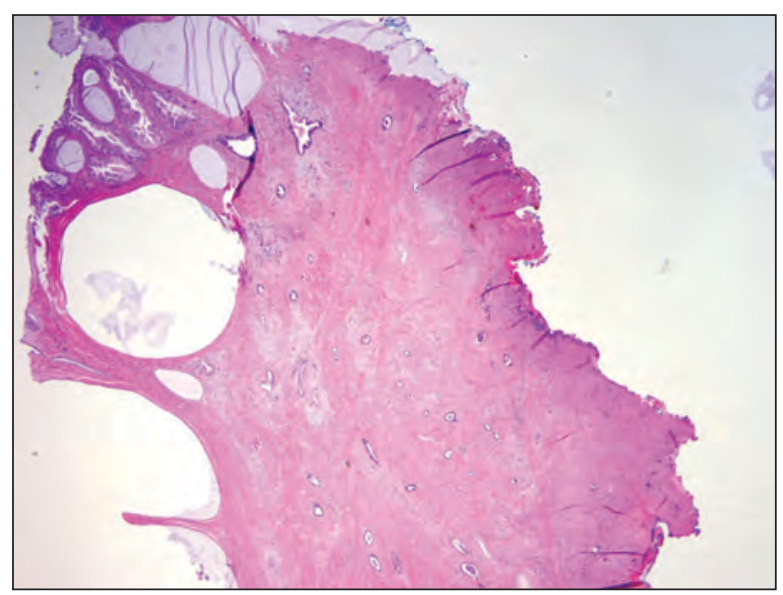

Figure 7. Gastric type endocervical adenocarcinoma. The low-power view reveals deep, destructive growth. (Olympus BX43, 2X). 


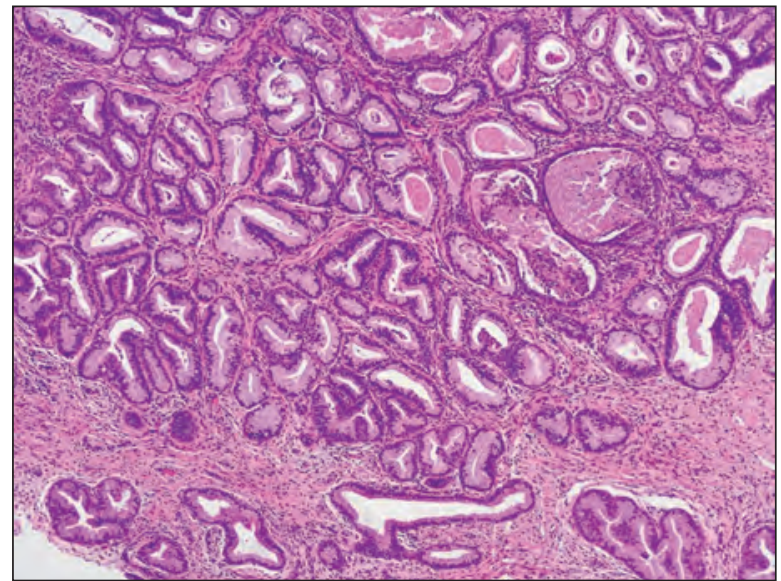

Figure 8. Complex glandular growth in gastric-type endocervical adenocarcinoma. Most cells are mucinous. (Olympus BX43, 10X).

\section{Clear Cell Type Endocervical Carcinoma}

Clear cell carcinoma of the lower genital tract has been historically connected with exposure to the diethylstilbestrol. Nowadays, this association is almost never seen. In current milieu of endocervical carcinomas, clear cell carcinoma is rather rare and usually presents in older patients. It is important to emphasize that it is not unheard of to see a younger patient present with clear cell carcinoma (Figure 10). The studies have shown that this carcinoma is not HPV-associated, and despite that about one-third of the cases have been reported to be p16 strongly positive. Positivity for p16 in these cases is seen as a result of aberrant Retinoblastoma $(R b)$ pathway rather than the HPV infection (17). Diagnosis is best achieved on morphologic criteria: large clear cells with abundant cytoplasm and hyalinized background. The nuclei are high-grade with prominent nucleoli. Immunohistochemistry is not particularly useful, but it is essential to mention that the cells are usually positive for HNF-1B, Napsin-A, MUC6 with sometimes aberrant p53 staining. The differential diagnosis in young patients includes pregnancy-related changes (AriasStella reaction) and exuberant microglandular hyperplasia (Figure 11) $(18,19)$. These benign glands are usually strongly positive for ER and PR, while clear cell carcinoma cells are usually negative.

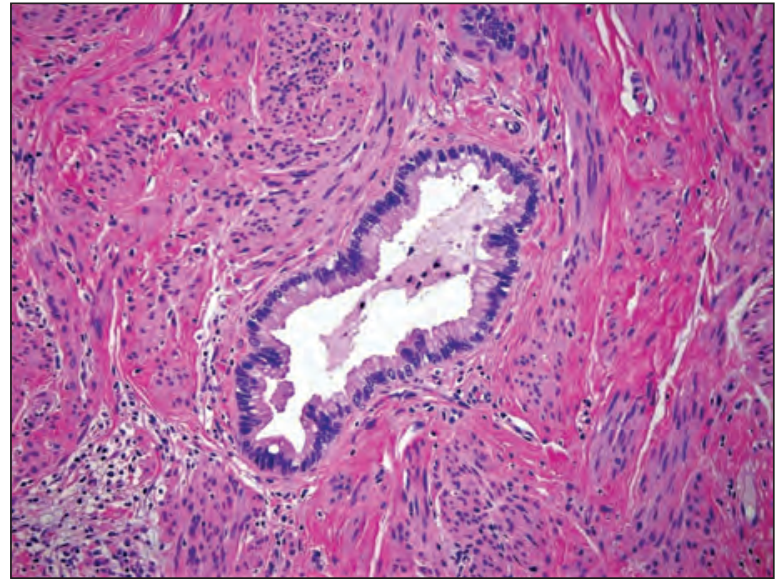

Figure 9. The glands may appear deceivingly bland and can be easily missed on the biopsy material. (Olympus BX43, 20x).

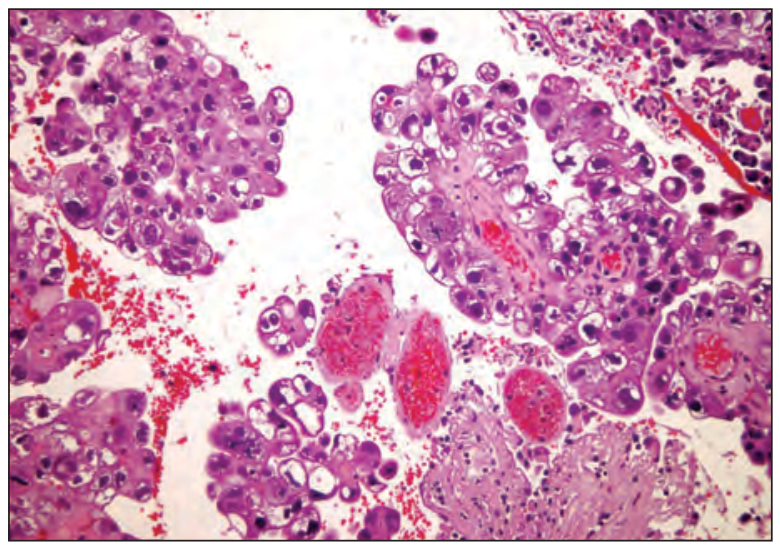

Figure 10. Large cervical mass in a young patient. The histologic finding of clear cell carcinoma. No history of exposure to the diethylstilbestrol. (Olympus BX43, 20x).

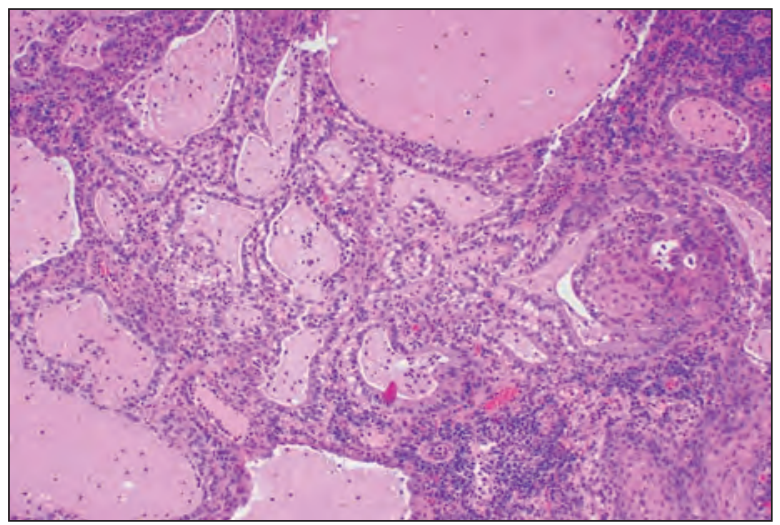

Figure 11. Microglandular adenosis/hyperplasia may look rather complex with cytoplasmic clearing. Nuclear features are low-grade with little variation in size. Mitoses are rare. (Olympus BX43, 10x). 


\section{Endometrioid Adenocarcinoma of Endocervix}

Endometrial adenocarcinoma of the cervix is a difficult entity to diagnose and the terminology may be confused with endometrial adenocarcinoma. Cervical primary tumor is associated with cervical endometriosis. Histologically, it exhibits endometrioid morphology, columnar pseudostratified cells with squamous morules with predominantly intermediate nuclear grade. This particular type of endocervical adenocarcinoma is rare and not HPV-associated (Figure 12). The primary consideration has to be given to the possibility of endometrial adenocarcinoma extending from the uterus. The best way to differentiate the two would be on hysterectomy specimens where the tumor must be extensively sampled, and the bulk of the tumor is arising from the cervix (13). Immunohistochemistry findings are similar to immunohistochemistry findings in classical endometrioid type adenocarcinoma with patchy 16 positivity, variable positivity for estrogen (ER) and progesterone $(\mathrm{PR})$, and a consistent vimentin positivity.

\section{Mesonephric-Type Adenocarcinoma}

This extremely rare tumor usually arises from mesonephric remnants. Mesonephric adenocarcinoma usually displays various growth patterns: ducts, tubules, papillary formations, and slit-like glands. One pattern can be dominant or solitary in the tumor. Cytologically, mesonephric carcinomas reveal bland tumor cells with scant cytoplasm with tubular structures with luminal eosinophilic secretions (Figure 13). Immunohistochemistry findings are extremely helpful in establishing the diagnosis. The tumors are usually negative for ER and PR but are GATA3, calretinin, CD10, and TTF-1 positive $(20,21)$. The molecular studies have shown alterations in KRAS and NRAS genes and chromatin remodeling gene mutations such as ARID1A, ARID1B, and SMARCA4 (22). The primary differential diagnosis is a florid type mesonephric hyperplasia (Figure 14).

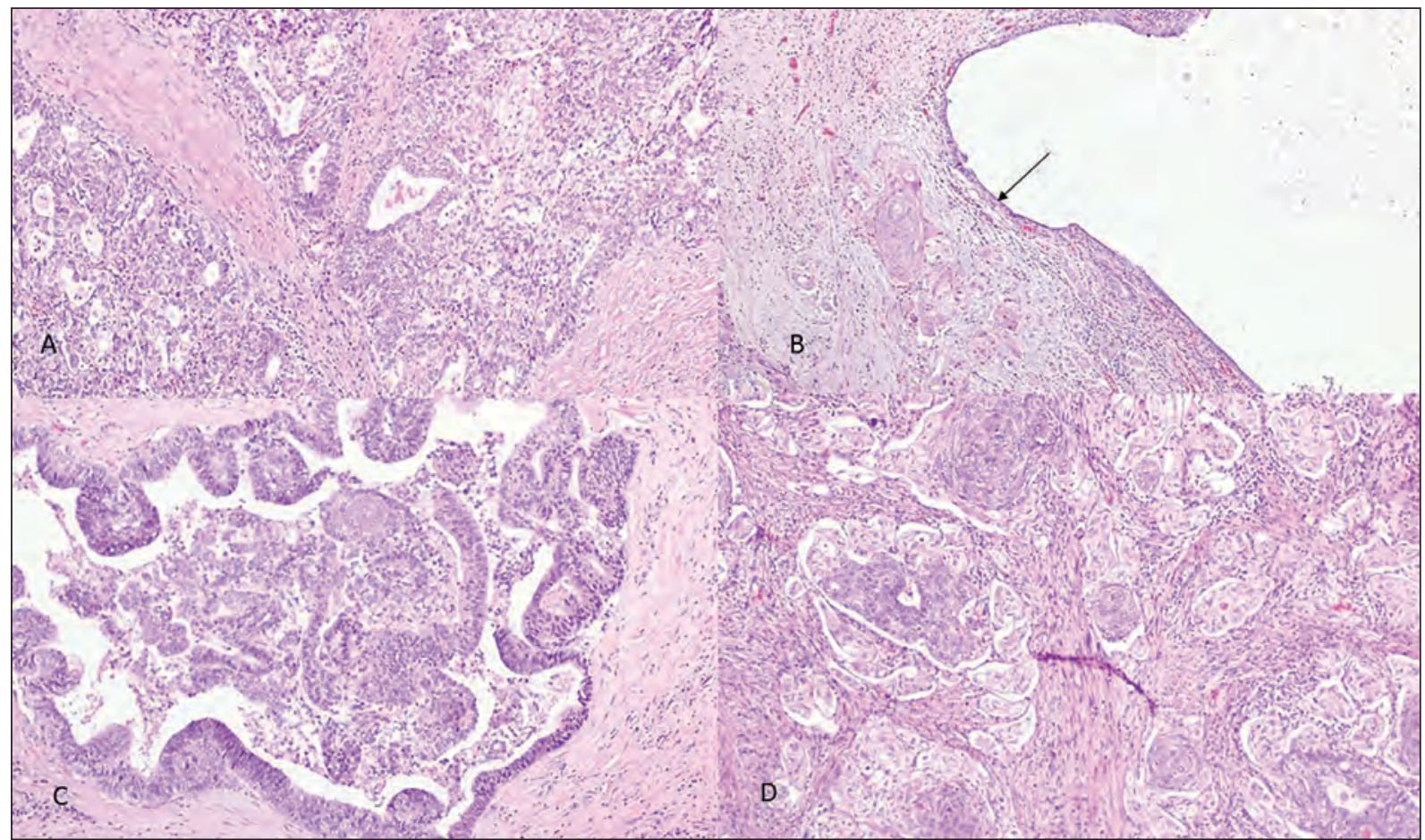

Figure 12A-D. A. Endometrioid adenocarcinoma of the uterine cervix with back to back glands B. Foci of endometriosis C. Focally more classical endometrioid cytologic features D. Prominent squamous morules. (Olympus BX43, A-10X, B-4X, C-20x, D-20x). 


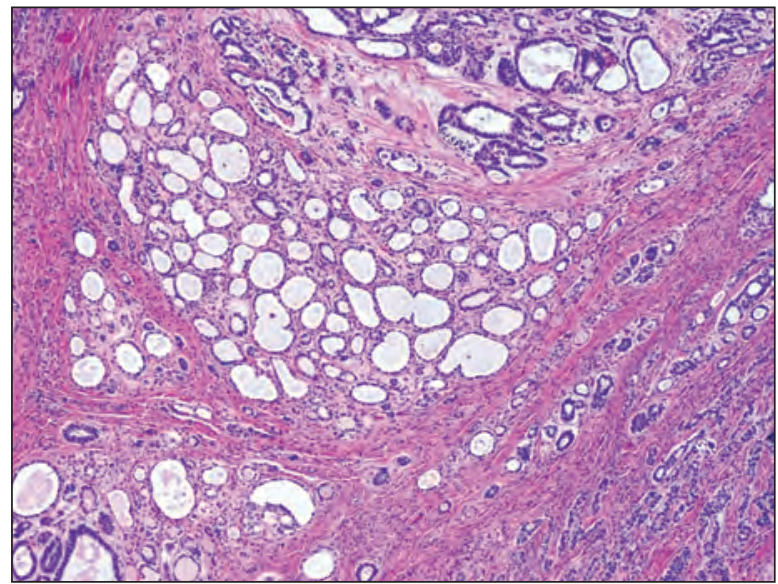

Figure 13. Mesonephric carcinoma of the cervix with infiltrative growth pattern composed of small tubes lined by cuboidal cells with scant cytoplasm. (Olympus BX43, 4X).

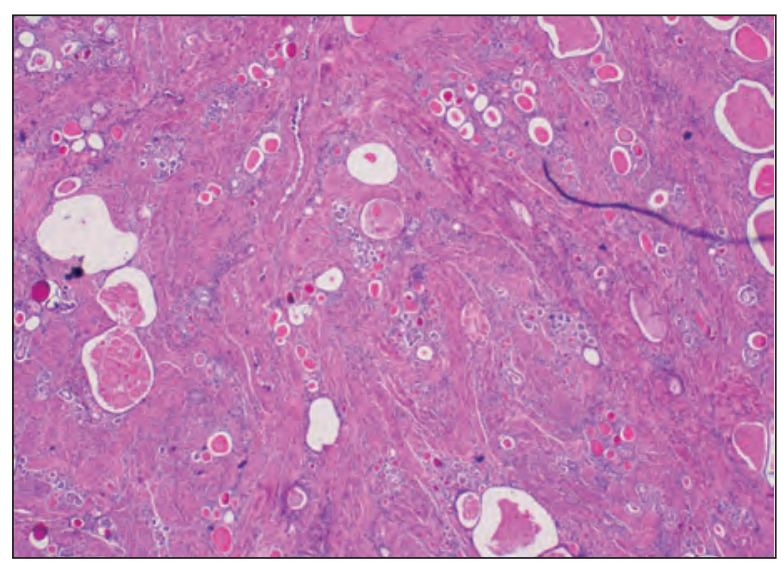

Figure 14. Florid mesonephric hyperplasia retains lobular architecture with associated ducts. No glandular crowding, solid growth, or nuclear atypia. (Olympus BX43, 4X).

\section{Pattern Based Classification of Endocervical Adenocarcinoma}

The pattern-based classification of endocervical adenocarcinoma (Silva pattern of invasion) was first recognized and published several years ago (23-26). Recently, the College of American Pathologists (CAP) included this classification as optional data into a synoptic report. Measuring the depth of invasion for endocervical adenocarcinoma can be problematic since it is difficult to estimate from which endocervical gland the invasion originates. Besides, there are no provided guidelines for measurements of the depth of invasion. Sometimes, endocervical adenocarcinoma grows exophytically, and it may be difficult to subtract exuberant exophytic growth from a true invasion. This may lead to an over-estimate of the depth of invasion, consequently causing overtreatment. The pattern-based classification approach has been proven to have quite good interobserver variability and an excellent prognostic value (23). It is important to mention that the system applies only to HVP-associated usual type endocervical adenocarcinoma. The entire tumor has to be examined microscopically to assign the appropriate group. This three-tier system essentially provides risk stratification analysis for the patients (24). The invasion and pattern-based classification was developed in 2013, and the system was validated in subsequent studies, including the assessment of interobserver variability (25-28).

Pattern $A$ can be assigned to the tumors composed of glands with rounded contours, frequently forming groups, and cribriform structures. Papillary growth is acceptable. No destructive stromal invasion, no single cells, or lymphovascular invasion should be seen in this pattern. The solid growth is not acceptable (Figures 15-16) $(26,27)$.

Pattern $\boldsymbol{B}$ can show focal destructive stromal invasion mostly from the pattern A appearing glands with small groups of tumor cells within desmoplastic stroma or inflammation. Invasion foci can be single, multiple, or linear at the tumor base. The lymphovascular invasion may be present. Solid growth is not acceptable (Figure 17) (26).

Pattern $C$ is easily recognized as a destructive stromal invasion with infiltrating angulated and open glands or solid growth pattern. It is important to remember that the solid growth pattern is considered a high-grade pattern, and nuclear grade can be disregarded. The extensive desmoplastic response is frequently present (Figures 1820). The lymphovascular invasion may be present.

It is important to emphasize that quantitative criteria for pattern $\mathrm{C}$ classification is a linear focus of destructive invasion at the base or advancing front of the tumor filling the diameter of $4 \mathrm{X}$ field ( 5 millimeters or more). This guideline is provided to give some quantitative guidelines to better distinction distinguish pattern $\mathrm{B}$ from pattern $\mathrm{C}$ classification (26). 


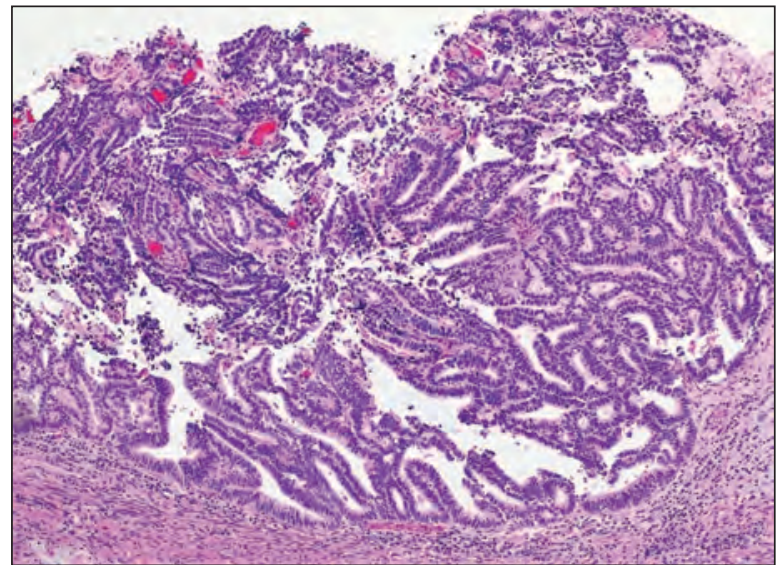

Figure 15. Back to back glands with rounded contours. This exuberant architectural complexity differentiates it from in-situ adenocarcinoma. No infiltrative or solid growth. The findings are compatible with pattern A tumor. (Olympus BX43, 10X).

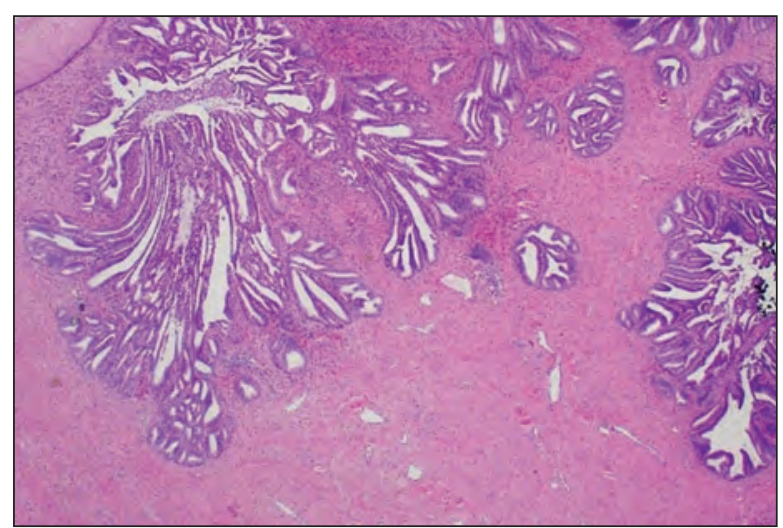

Figure 16. Low power view of pattern A tumor; deeper portions of the tumor do not elicit a stromal response. (Olympus BX43, 4X).

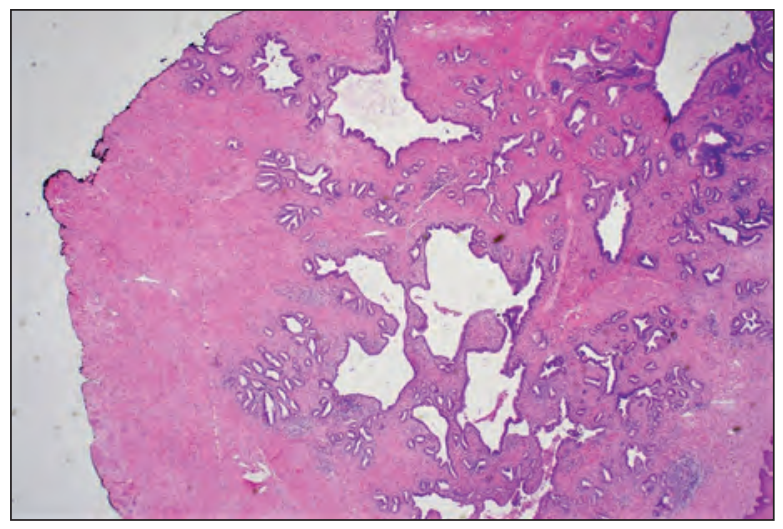

Figure 17. Pattern B shows predominately pattern A glands with foci of destructive growth. This emphasizes the importance of examining the entire specimen before assigning the pattern. (Olympus BX43, 4X).

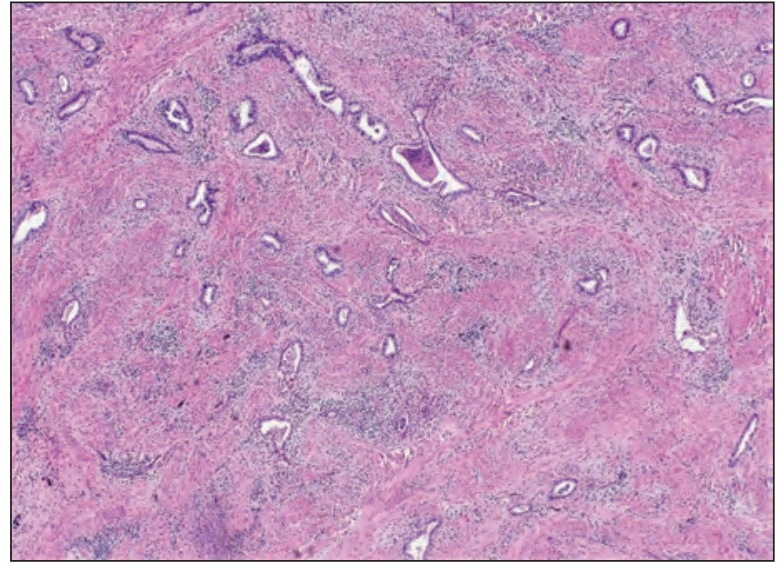

Figure 18. This tumor was assigned pattern $C$ with deep invasion and infiltrative growth. (Olympus BX43, 4X).

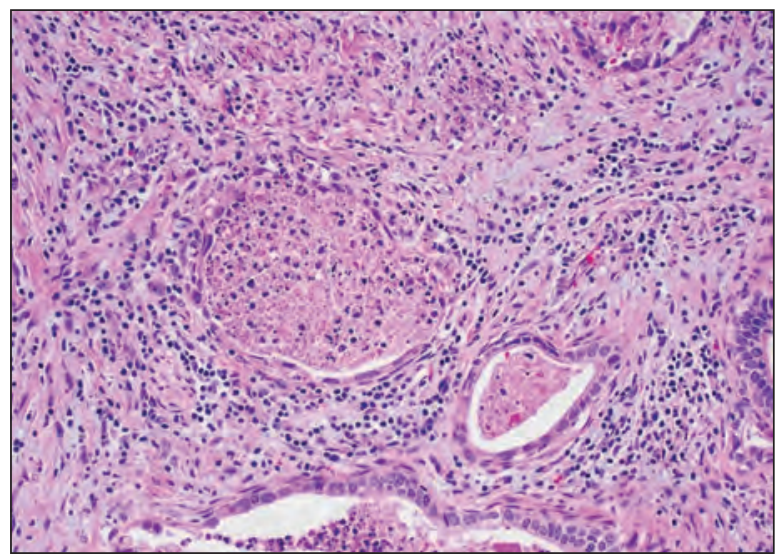

Figure 19. High-power view of desmoplastic response and inflammation around every gland. (Olympus BX43, 40X).

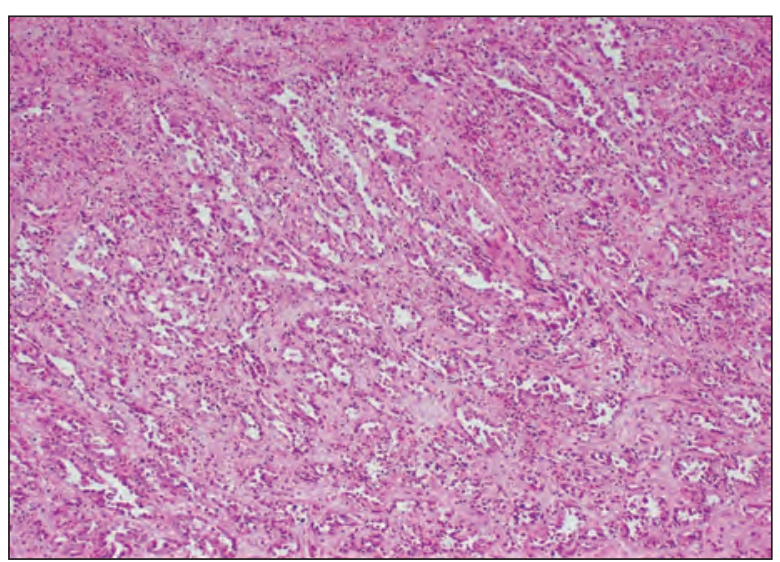

Figure 20. Another pattern $C$ destructive growth and mazelike glands. (Olympus BX43, 20x). 


\section{Risk Stratification and Clinical Outcomes}

Tumors classified as pattern A are not associated with a risk of lymph node metastasis. They are limited to stage one, and they do not recur. Pattern B tumors also frequently present as stage one. However, given the allowed presence of lymphovascular space invasion, some tumors are at risk for lymph node metastasis. The studies have shown that the pattern $\mathrm{C}$ tumors frequently present at the higher stage, and $22 \%$ of tumors present with lymph node metastasis. Additionally, about $20 \%$ of the cases show a local recurrence of the tumor (28). The classification is useful and reproducible only in excision procedures, and it has limited potential in biopsy material, particularly in pattern A tumors. However, based on the presence of lymphovascular space invasion or solid growth pattern in the biopsy material, the patient may undergo treatment with a more aggressive deeper excision instead of a superficial loop electrosurgical excision procedure (LEEP) $(29,30)$.

\section{FIGO Staging}

The International Federation of Gynecology and Obstetrics (FIGO) staging system is based on the presence or absence of a clinically visible or palpable lesion. The clinically visible mass implies at least stage FIGO IB1. If the tumor is not clinically visible, the staging relies on microscopic measurements. IA1 includes $\leq 3 \mathrm{~mm}$ depth, whereas IA2 includes $>3 \mathrm{~mm}$ but $<5 \mathrm{~mm}$ depth. Horizontal extent is now an optional data point in CAP synoptic report. It is no longer used in the AJCC staging update of the 2018 FIGO classification (31). However, the pathologists are encouraged to report horizontal spread in smaller tumors since it is still a valuable data point for future research.

\section{Conclusions}

In conclusion, International Endocervical Adenocarcinoma Criteria and Classification (IECC) is a simple classification system that recognizes and classifies endocervical tumors based on pathogen- esis and association to HPV. This may facilitate better treatment options based on the natural history of this cancer. The pathologists should also be familiar with the pattern-based classification of endocervical adenocarcinoma. If excisional biopsies show pattern A or B, the entire tumor should be submitted. Future treatments can be based on this classification system, given an excellent interobserver variability. Lastly, FIGO staging does not include horizontal extent, but the pathologists are encouraged to provide this optional data point for future research purposes.

Conflict of Interest: The authors declare that they have no conflict of interest.

\section{References}

1. Wilbur DC, Colgan TJ, Ferenczy AS, Hirschowitz L, Loening $\mathrm{T}, \mathrm{McCl}$ cluggage WG, et al. Tumors of the uterine cervix - glandular tumors and precursors. In: Kurman RJ, Carcangiu ML, Herrington CS, Young RH, editors. WHO classification of tumours of female reproductive organs. 4th edition. Lyon: International Agency for Research on Cancer; 2014. p. 183-94.

2. Kurman RJ, Carcangiu ML, Herrington CS, Young RH, editors. WHO classification of tumors of female reproductive organs. 4th edition. Lyon: International Agency for Research on Cancer; 2014.

3. Stolnicu S, Barsan I, Hoang L, Patel P, Terinte C, Pesci A, et al. International Endocervical Adenocarcinoma Criteria and Classification (IECC): A New Pathogenetic Classification for Invasive Adenocarcinomas of the Endocervix. Am J Surg Pathol. 2018;42(2):214-26.

4. Pirog EC, Lloveras B, Molijn A, Tous S, Guimerà N, Alejo $\mathrm{M}$, et al. HPV prevalence and genotypes in different histological subtypes of cervical adenocarcinoma, a worldwide analysis of 760 cases. Mod Pathol. 2014;27(12):1559-67.

5. Stolnicu S, Hoang L, Soslow RA. Recent advances in invasive adenocarcinoma of the cervix. Virchows Arch. 2019;475(5):537-49.

6. Korach J, Machtinger R, Perri T, Vicus D, Segal J, Fridman E, et al. Villoglandular papillary adenocarcinoma of the uterine cervix: a diagnostic challenge. Acta Obstet Gynecol Scand. 2009;88(3):355-8.

7. Jones MW, Silverberg SG, Kurman RJ. Well-differentiated villoglandular adenocarcinoma of the uterine cervix: a clinicopathological study of 24 cases. Int J Gynecol Pathol. 1993;12(1):1-7.

8. Stewart CJR, Koay MHE, Leslie C, Acott N, Leung YC. Cervical carcinomas with a micropapillary component: a 
clinicopathological study of eight cases. Histopathology. 2018;72(4):626-33.

9. Toyoda S, Kita T, Sugiura A, Itani Y, Okada H, Nakamura $\mathrm{S}$, et al. Cervical adenocarcinoma with stromal micropapillary pattern. Diagn Cytopathol. 2016;44(2):133-6.

10. Alvarado-Cabrero I, McCluggage WG, Estevez-Castro R, Pérez-Montiel D, Stolnicu S, Ganesan R, et al. Micropapillary Cervical Adenocarcinoma: A Clinicopathologic Study of 44 Cases. Am J Surg Pathol. 2019;43(6):802-9.

11. Lastra RR, Park KJ, Schoolmeester JK. Invasive Stratified Mucin-producing Carcinoma and Stratified Mucin-producing Intraepithelial Lesion (SMILE): 15 Cases Presenting a Spectrum of Cervical Neoplasia With Description of a Distinctive Variant of Invasive Adenocarcinoma. Am J Surg Pathol. 2016;40(2):262-9.

12. Stolnicu S, Hoang L, Hanko-Bauer O, Barsan I, Terinte C, Pesci A, et al. Cervical adenosquamous carcinoma: detailed analysis of morphology, immunohistochemical profile, and clinical outcomes in 59 cases. Mod Pathol. 2019;32(2):269-79.

13. Stolnicu S, Hoang L, Soslow RA. Recent advances in invasive adenocarcinoma of the cervix. Virchows Arch. 2019;75(5):537-49.

14. Park E, Kim SW, Kim S, Kim HS, Lee JY, Kim YT, et al. Genetic characteristics of gastric-type mucinous carcinoma of the uterine cervix. Mod Pathol. 2020 Jul 8. doi: 10.1038/s41379-020-0614-0. Epub ahead of print.

15. Moat M, O'Donnell RL, McCluggage WG, Ralte A, Edmondson RJ. Gastric-type adenocarcinoma of the cervix in a patient with Lynch syndrome: A case report. Gynecol Oncol Rep. 2014;10:41-3.

16. Ito M, Minamiguchi S, Mikami Y, Ueda Y, Sekiyama K, Yamamoto T, et al. Peutz-Jeghers syndrome-associated atypical mucinous proliferation of the uterine cervix: a case of minimal deviation adenocarcinoma ('adenoma malignum') in situ. Pathol Res Pract. 2012;208(10):623-7.

17. Stolnicu S, Barsan I, Hoang L, Patel P, Chiriboga L, Terinte C, et al. Diagnostic Algorithmic Proposal Based on Comprehensive Immunohistochemical Evaluation of 297 Invasive Endocervical Adenocarcinomas. Am J Surg Pathol. 2018;42(8):989-1000.

18. Nucci MR, Young RH. Arias-Stella reaction of the endocervix: a report of 18 cases with emphasis on its varied histology and differential diagnosis. Am J Surg Pathol. 2004;28(5):608-12.

19. Nucci, M. Pseudoneoplastic Glandular Lesions of the Uterine Cervix: A Selective Review. Int J Gynecol Pathol. 2014;33(4):330-8.

20. Silver SA, Devouassoux-Shisheboran M, Mezzetti TP, Tavassoli FA. Mesonephric adenocarcinomas of the uterine cervix: a study of 11 cases with immunohistochemical findings. Am J Surg Pathol. 2001;25(3):379-87.
21. Kenny SL, McBride HA, Jamison J, McCluggage WG. Mesonephric adenocarcinomas of the uterine cervix and corpus: HPV-negative neoplasms that are commonly PAX8, CA125, and HMGA2 positive and that may be immunoreactive with TTF1 and hepatocyte nuclear factor $1-\beta$. Am J Surg Pathol. 2012;36(6):799-807.

22. Mirkovic J, Sholl LM, Garcia E, Lindeman N, MacConaill L, Hirsch M, et al. Targeted genomic profiling reveals recurrent KRAS mutations and gain of chromosome $1 \mathrm{q}$ in mesonephric carcinomas of the female genital tract. Mod Pathol. 2015;28(11):1504-14.

23. Rutgers JK, Roma AA, Park KJ, Zaino RJ, Johnson A, Alvarado I, et al. Pattern classification of endocervical adenocarcinoma: reproducibility and review of criteria. Mod Pathol. 2016;29(9):1083-94.

24. Turashvili G, Park KJ. Cervical Glandular Neoplasia: Classification and Staging. Surg Pathol Clin. 2019;12(2):281313.

25. Diaz De Vivar A, Roma AA, Park KJ, Alvarado-Cabrero I, Rasty G, Chanona-Vilchis JG, et al. Invasive endocervical adenocarcinoma: proposal for a new pattern-based classification system with significant clinical implications: a multi-institutional study. Int J Gynecol Pathol. 2013;32(6):592-601

26. Roma AA, Diaz De Vivar A, Park KJ, Alvarado-Cabrero I, Rasty G, Chanona-Vilchis JG, et al. Invasive endocervical adenocarcinoma: a new pattern-based classification system with important clinical significance. Am J Surg Pathol. 2015;39(5):67-72.

27. Paquette C, Jeffus SK, Quick CM, Conaway MR, Stoler $\mathrm{MH}$, Atkins KA. Interobserver variability in the application of a proposed histologic subclassification of endocervical adenocarcinoma. Am J Surg Pathol. 2015;39(1):93100 .

28. Roma AA, Park KJ, Xie H, De Vivar AD, Alvarado-Cabrero I, Rutgers JKL, et al. Role of Lymphovascular Invasion in Pattern C Invasive Endocervical Adenocarcinoma. Am J Surg Pathol. 2017;41(9):1205-11.

29. Roma AA, Mistretta TA, Diaz De Vivar A, Park KJ, Alvarado-Cabrero I, Rasty G, et al. New pattern-based personalized risk stratification system for endocervical adenocarcinoma with important clinical implications and surgical outcome. Gynecol Oncol. 2016;141(1):36-42.

30. Roma AA, Diaz De Vivar A, Park KJ, Alvarado-Cabrero I, Rasty G, Chanona-Vilchis JG, et al. Invasive endocervical adenocarcinoma: a new pattern-based classification system with important clinical significance. Am J Surg Pathol. 2015;39(5):667-72.

31. Bhatla N, Berek JS, Cuello Fredes M, Denny LA, Grenman S, Karunaratne K, et al. Revised FIGO staging for carcinoma of the cervix uteri. Int J Gynaecol Obstet. 2019;145(1):129-35 